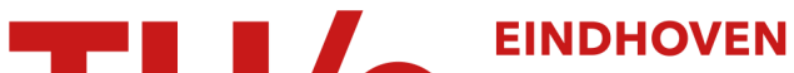 UNIVERSITY OF TECHNOLOGY
}

\section{Direct identification of continuous-time LPV models}

Citation for published version (APA):

Laurain, V., Gilson, M., Toth, R., \& Garnier, H. (2011). Direct identification of continuous-time LPV models. In Proceedings of the American Control Conference, 29 June-1 July 2011, San Francisco, California (pp. 159-164)

Document status and date:

Published: 01/01/2011

\section{Document Version:}

Accepted manuscript including changes made at the peer-review stage

\section{Please check the document version of this publication:}

- A submitted manuscript is the version of the article upon submission and before peer-review. There can be important differences between the submitted version and the official published version of record. People interested in the research are advised to contact the author for the final version of the publication, or visit the $\mathrm{DOI}$ to the publisher's website.

- The final author version and the galley proof are versions of the publication after peer review.

- The final published version features the final layout of the paper including the volume, issue and page numbers.

Link to publication

\section{General rights}

Copyright and moral rights for the publications made accessible in the public portal are retained by the authors and/or other copyright owners and it is a condition of accessing publications that users recognise and abide by the legal requirements associated with these rights.

- Users may download and print one copy of any publication from the public portal for the purpose of private study or research.

- You may not further distribute the material or use it for any profit-making activity or commercial gain

- You may freely distribute the URL identifying the publication in the public portal.

If the publication is distributed under the terms of Article 25fa of the Dutch Copyright Act, indicated by the "Taverne" license above, please follow below link for the End User Agreement:

www.tue.nl/taverne

Take down policy

If you believe that this document breaches copyright please contact us at:

openaccess@tue.nl

providing details and we will investigate your claim. 


\title{
Direct identification of continuous-time LPV models
}

\author{
V. Laurain*, M. Gilson*, R. Tóth**, H. Garnier*
}

\begin{abstract}
Controllers in the linear parameter-varying (LPV) framework are commonly designed in continuous time (CT) requiring accurate and low-order CT models of the system. Nonetheless, most of the methods dedicated to the identification of LPV systems are addressed in the discrete-time setting. In practice when discretizing models which are naturally expressed in $\mathrm{CT}$, the dependency on the scheduling variables becomes non-trivial and over-parameterized. Consequently, direct identification of CT-LPV systems in an input-output setting is investigated. To provide consistent model parameter estimates in this setting, a refined instrumental variable approach is proposed. The statistical properties of this approach are demonstrated through a Monte Carlo simulation example.
\end{abstract}

\section{INTRODUCTION}

The framework of linear parameter-varying (LPV) systems was introduced in the 1990s with the purpose to handle in a simple but efficient way the often nonlinear or timevarying nature of systems encountered in practice. The LPV system class forms an intermediate step between linear timeinvariant (LTI) systems and nonlinear/time-varying plants as the signal relations in LPV systems are considered to be linear just as in the LTI case, but the parameters are assumed to be functions of a measurable time-varying signal, the socalled scheduling variable $p: \mathbb{Z} \rightarrow \mathbb{P}$. Here the compact set $\mathbb{P} \subset \mathbb{R}^{n_{\mathbb{P}}}$ denotes the scheduling space. This LPV modeling concept allows for a wide representation capability of physical processes, but the real practical significance of the LPV framework lays in its well worked out and industrially reputed control synthesis approaches, e.g. [1], [18], [24], that have led to many successful applications of LPV control in practice [3], [13], [14], [23].

However a major drawback of the LPV framework today is that, despite the advances of the LPV control field, identification of such systems is not well developed as the current methods are unable to support control design in practice. Commonly LPV controllers are synthesized in continuous time (CT) as stability and performance requirements of the closed loop behavior can be more conveniently expressed in CT, like in a mixed-sensitivity setting [28].

However, LPV identification methods are almost exclusively developed for discrete time (DT) (for a recent survey see [20]), as in this setting it is much easier to handle the estimation of parameter-varying dynamics. Nonetheless,

\footnotetext{
* Centre de Recherche en Automatique de Nancy (CRAN), Nancyuniversité, CNRS, BP 239, 54506 Vandoeuvre-les-Nancy Cedex, France, vincent. laurain@cran. uhp-nancy. fr.

**Delft Center for Systems and Control (DCSC), Delft University of Technology, Mekelweg 2, 2628 CD Delft, The Netherlands, r.tothetudelft.nl. Support by the Netherlands Organization for Scientific Research (NWO) is gratefully acknowledged (grant no. 680-500927).
}

the absence of CT methods represents a gap between the available identification approaches and the needs of LPV control synthesis.

Therefore, there is a growing need in the LPV framework for efficient identification methods that directly deliver reliable CT models.

In practice, CT systems can only be identified based on sampled measured data records. Thus in general, for delivering a CT model estimate, the available approaches in system identification can be categorized as follows:

- Indirect approaches: These methods involve the identification of a DT model in a completely DT setting which is followed by the transformation of the DT model estimate into a CT form.

- Direct approaches: The methods formulate the identification of the CT model directly based on samples of the measured CT signals.

Unfortunately, transformation of DT-LPV models to CTLPV models is more complicated than in the LTI case and despite recent advances in LPV discretization theory (see [21], [22]) the CT realization of DT models is still in an immature state. LPV discretization can result in an increase of the system order and more importantly in complicated (rational) dynamic dependence on $p$ (dependence of the model coefficients on time-shifted versions of $p$ ) for which most of the available identification methods are not well suited. An illustrative example is the estimation of an LPV longitudinal axis IO model of an aircraft where the coefficients associated with the stability (output-side) and control derivatives (input-side) relate rationally to the DT parameters even if only a successive Euler method is used to approximate the derivatives. Even for a simple CT-LPV system, estimation of a DT model to obtain afterwards a CT realization is a tedious task with many underlaying problems for which there are no general theoretical solutions available.

Unlike an indirect approach, a direct solution offers a way to efficiently overcome these problems in case the timederivatives of the input/output signals can be reconstructed from the acquired sampled data. The offered solutions in the LTI case often require the use of signals prefiltering [4]. These filters depend on design parameters, that need to be adequately chosen w.r.t. the underlaying unknown system to achieve reasonable performance of the estimation. In the CT-LTI case, one of the methods for relaxing the need of prefiltering is the refined instrumental variable for continuous-time (RIVC) method. This method is attractive in the sense that it provides consistent estimates under the realistic assumption of an unknown noise model and it achieves 
similar performance as prediction-error-minimization (PEM) methods [17].

Another problem related to CT identification using sampled data is the complicated mathematical handling of CT stochastic processes for modeling noise in the system. An efficient way to avoid such complexity is to consider a DT form of the noise model, like in a Box-Jenkins form, which leads to a so-called hybrid model structure (see [7], [15]). In order to avoid the different issues linked to the simultaneous use of CT and DT filters, this paper focuses on the case when the noise is output-additive and white with a Gaussian distribution: the output error (OE) case.

Recently, an LPV identification approach has been introduced, which efficiently addresses the minimization of the prediction error for LPV-OE models by using a multipleinput single-output (MISO) LTI reformulation of the datagenerating LPV system [9]. This paper aims at providing the very first step towards bridging the existing gap between LPV control and identification via the introduction of a direct CT identification approach. This method benefits from the properties of RIV methods in the CT case and uses the recent advances of the prediction error minimization framework [9], [20].

The paper is organized as follows: in Section II, the general class of CT-LPV systems in an IO representation form is introduced. In Section III, the proposed CT-LPV-IV method is described and analyzed, while its performance is illustrated in Section IV through a simulation example.

\section{PROBLEM DESCRIPTION}

\section{A. System description}

Consider the data generating CT-LPV system described by the following equations

$$
\mathcal{S}_{\mathrm{o}}\left\{\begin{array}{l}
A_{\mathrm{o}}\left(p_{t}, d\right) \chi_{\mathrm{o}}(t)=B_{\mathrm{o}}\left(p_{t}, d\right) u(t), \\
y(t)=\chi_{\mathrm{o}}(t)+e_{\mathrm{o}}(t),
\end{array}\right.
$$

where $d$ denotes the differentiation operator w.r.t. time, i.e. $d=\frac{\mathrm{d}}{\mathrm{d} t}, p: \mathbb{R} \rightarrow \mathbb{P}$ is the scheduling variable with $p_{t}=p(t)$, $\chi_{\mathrm{o}}$ is the noise-free output and $e_{\mathrm{o}}$ is a white gaussian noise process with variance $\sigma_{e_{\mathrm{o}}}^{2} . A_{\mathrm{o}}, B_{\mathrm{o}}$ are polynomials in $d$ with coefficients $a_{i}^{\mathrm{o}}$ and $b_{i}^{\mathrm{o}}$ that are meromorphic functions ${ }^{1}$ of $p$ with no singularity on $\mathbb{P}$ :

$$
\begin{aligned}
& A_{\mathrm{o}}\left(p_{t}, d\right)=d^{n_{\mathrm{a}}}+\sum_{i=1}^{n_{\mathrm{a}}} a_{i}^{\mathrm{o}}\left(p_{t}\right) d^{n_{\mathrm{a}}-i} \\
& B_{\mathrm{o}}\left(p_{t}, d\right)=\sum_{j=0}^{n_{\mathrm{b}}} b_{j}^{\mathrm{o}}\left(p_{t}\right) d^{n_{\mathrm{b}}-j}
\end{aligned}
$$

Note that $a_{i}^{\mathrm{o}}$ and $b_{j}^{\mathrm{o}}$ are functions of $p$ at time $t$, which is called static dependence. In LPV system theory, a more general $p$-dependence of coefficients than static is required to establish equivalence of representations. In particular, it can be required that the coefficients $a_{i}^{\mathrm{o}}$ and $b_{j}^{\mathrm{o}}$ depend also on time derivatives of $p$, which is called dynamic dependence [20]. In order to simplify the upcoming discussion,

\footnotetext{
${ }^{1}$ A function $f$ is called meromorphic if $f=\frac{g}{h}$ where $g, h$ are holomorphic (analytic) functions and $h$ is not the zero function.
}

we restrict our attention to static dependence. Nevertheless, the established results hold also in the case of dynamic dependence of (1) and of the proposed model structure.

In terms of identification we can assume that sampled measurements of $(y, p, u)$ are available with a sampling period $\mathrm{T}_{\mathrm{S}}>0$. Hence, we will denote the discrete-time samples of these signals as $u\left(t_{k}\right)=u\left(k \mathrm{~T}_{\mathrm{s}}\right)$, where $k \in \mathbb{Z}$.

\section{B. Model structure considered}

The process model is denoted by $\mathcal{G}_{\rho}$ and defined in a form of an LPV-IO representation with a static scheduling dependence:

$$
\mathcal{G}_{\rho}:\left(A\left(p_{t}, d, \rho\right), B\left(p_{t}, d, \rho\right)\right)
$$

where the $p$-dependent polynomials $A$ and $B$ given as

$$
\begin{aligned}
& A\left(p_{t}, d, \rho\right)=d^{n_{\mathrm{a}}}+\sum_{i=1}^{n_{\mathrm{a}}} a_{i}\left(p_{t}\right) d^{n_{\mathrm{a}}-i}, \\
& B\left(p_{t}, d, \rho\right)=\sum_{j=0}^{n_{\mathrm{b}}} b_{j}\left(p_{t}\right) d^{n_{\mathrm{b}}-j},
\end{aligned}
$$

are parameterized as

$$
\begin{aligned}
& a_{i}\left(p_{t}\right)=a_{i, 0}+\sum_{l=1}^{n_{\alpha}} a_{i, l} f_{l}\left(p_{t}\right), \quad i=1, \ldots, n_{\mathrm{a}} \\
& b_{j}\left(p_{t}\right)=b_{j, 0}+\sum_{l=1}^{n_{\beta}} b_{j, l} g_{l}\left(p_{t}\right), \quad j=0, \ldots, n_{\mathrm{b}}
\end{aligned}
$$

In this parametrization, $\left\{f_{l}\right\}_{l=1}^{n_{\alpha}}$ and $\left\{g_{l}\right\}_{l=1}^{n_{\beta}}$ are meromorphic functions of $p$, with static dependence, allowing the identifiability of the model (they can be chosen for example as linearly independent functions on $\mathbb{P}$ ). The associated model parameters are stacked column-wise:

$$
\rho=\left[\begin{array}{llllll}
\mathrm{a}_{1} & \ldots & \mathrm{a}_{n_{\mathrm{a}}} & \mathrm{b}_{0} & \ldots & \mathrm{b}_{n_{\mathrm{b}}}
\end{array}\right]^{\top} \in \mathbb{R}^{n_{\rho}},
$$

where

$$
\begin{aligned}
\mathrm{a}_{i} & =\left[\begin{array}{llll}
a_{i, 0} & a_{i, 1} & \ldots & a_{i, n_{\alpha}}
\end{array}\right] \in \mathbb{R}^{n_{\alpha}+1} \\
\mathrm{~b}_{j} & =\left[\begin{array}{llll}
b_{j, 0} & b_{j, 1} & \ldots & b_{j, n_{\beta}}
\end{array}\right] \in \mathbb{R}^{n_{\beta}+1}
\end{aligned}
$$

and $n_{\rho}=n_{\mathrm{a}}\left(n_{\alpha}+1\right)+\left(n_{\mathrm{b}}+1\right)\left(n_{\beta}+1\right)$. Introduce also $\mathcal{G}=\left\{\mathcal{G}_{\rho} \mid \rho \in \mathbb{R}^{n_{\rho}}\right\}$, as the collection of all process models in the form of (3).

With respect to the considered OE structure, the signal relations of the LPV-BJ model, denoted in the sequel as $\mathcal{M}_{\rho}$, are defined as:

$$
\mathcal{M}_{\rho}\left\{\begin{array}{l}
A\left(p_{k}, d, \rho\right) \chi(t)=B\left(p_{k}, d, \rho\right) u(t), \\
y\left(t_{k}\right)=\chi\left(t_{k}\right)+e\left(t_{k}\right),
\end{array}\right.
$$

Based on this model structure, the model set, denoted as $\mathcal{M}=\left\{\mathcal{M} \rho \mid \rho \in \mathbb{R}^{n_{\rho}}\right\}=\mathcal{G}$, corresponds to the set of candidate models in which we seek the model that explains data gathered from $\mathcal{S}_{\mathrm{o}}$ the best, under a given identification criterion (cost function). 


\section{Predictors and prediction error}

Similar to the LTI case, in the LPV prediction error framework, one is concerned about finding a model in a given LPV model structure $\mathcal{M}$, which minimizes the statistical mean of the squared prediction error based on past samples of $(y, u, p)$. However in the LPV case, no transfer function representation of systems is available.

Furthermore, multiplication with $d$ is not commutative over the $p$-dependent coefficients [20], meaning that

$$
\begin{array}{r}
d(B(p, d) u(t))=\sum_{j=0}^{n_{\mathrm{b}}}\left(\frac{\partial b_{j}}{\partial p}\left(p_{t}\right) d p_{t}\right) d^{n_{\mathrm{b}}-j} u(t)+ \\
B(p, d) d u(t),
\end{array}
$$

which is not equal to $B(p, d) d u(t)$.

1) System reformulation and prediction error: Following the same idea developed in [9] and if the system belongs to the model set defined, it is possible to express the CT-LPV system as a CT-MISO-LTI system by rewriting the signal relations of (1) as

$$
\begin{gathered}
\underbrace{\chi_{\mathrm{o}}^{\left(n_{\mathrm{a}}\right)}(t)+\sum_{i=1}^{n_{\mathrm{a}}} a_{i, 0}^{\mathrm{o}} \chi_{\mathrm{o}}^{\left(n_{\mathrm{a}}-i\right)}(t)}_{F_{\mathrm{o}(d)} \chi_{\mathrm{o}}(t)}+\sum_{i=1}^{n_{\mathrm{a}}} \sum_{l=1}^{n_{\alpha}} a_{i, l}^{\mathrm{o}} \underbrace{f_{l}(p(t)) \chi_{\mathrm{o}}^{\left(n_{\mathrm{a}}-i\right)}(t)}_{\chi_{i, l}^{\mathrm{o}}(t)} \\
=\sum_{j=0}^{n_{\mathrm{b}}} \sum_{l=0}^{n_{\beta}} b_{j, l}^{\mathrm{o}} \underbrace{g_{l}(p(t)) u^{\left(n_{\mathrm{b}}-j\right)}(t)}_{u_{j, l}(t)},
\end{gathered}
$$

where $g_{0}(\cdot) \equiv 1$ and the superscript $(n)$ for a signal, like $u^{(n)}$, denotes the $n^{\text {th }}$ time-derivative of the signal, e.g. $u^{(n)}(t)=d^{n} u(t)$. Furthermore, $F_{\mathrm{o}}(d)=d^{n_{\mathrm{a}}}+$ $\sum_{i=1}^{n_{\mathrm{a}}} a_{i, 0}{ }^{n_{\mathrm{a}}-i}$ while $u^{(n)}\left(t_{k}\right)$ represents the value of the signal $u^{(n)}(t)$ sampled at time instance $t_{k}$.

Note that in this way, the time variation of the coefficients is expressed in terms the signals $\chi_{i, l}^{\circ}(t)$ and $u_{j, l}(t)$ :

$$
\begin{array}{ll}
\chi_{i, l}^{\mathrm{o}}(t)=f_{l}(p(t)) \chi_{\mathrm{o}}^{\left(n_{\mathrm{a}}-i\right)}(t) & \{i, l\} \in\left\{1 \ldots n_{\mathrm{a}}, 1 \ldots n_{\alpha}\right\}, \\
u_{j, l}(t)=g_{l}(p(t)) u^{\left(n_{\mathrm{b}}-j\right)}(t) & \{j, l\} \in\left\{1 \ldots n_{\mathrm{b}}, 1 \ldots n_{\alpha}\right\} .
\end{array}
$$

Therefore, the process part of the LPV-BJ model is rewritten as a MISO system with $\left(n_{\mathrm{b}}+1\right)\left(n_{\beta}+1\right)+n_{\mathrm{a}} n_{\alpha}$ inputs $\left\{\chi_{i, l}^{\mathrm{o}}\right\}_{i=1, l=1}^{n_{\mathrm{a}}, n_{\alpha}}$ and $\left\{u_{j, l}\right\}_{j=0, l=0}^{n_{\mathrm{b}}, n_{\beta}}$. By using (8), (6) can be rewritten in terms of the sampled output signal $y\left(t_{k}\right)$ as

$$
\begin{aligned}
y\left(t_{k}\right) & =-\left(\sum_{i=1}^{n_{\mathrm{a}}} \sum_{l=1}^{n_{\alpha}} \frac{a_{i, l}^{\mathrm{o}}}{F_{\mathrm{o}}(d)} \chi_{i, l}^{\mathrm{o}}\right)\left(t_{k}\right) \\
& +\left(\sum_{j=0}^{n_{\mathrm{b}}} \sum_{l=0}^{n_{\beta}} \frac{b_{j, l}^{\mathrm{o}}}{F_{\mathrm{o}}(d)} u_{k, j}\right)\left(t_{k}\right)+e_{\mathrm{o}}\left(t_{k}\right),
\end{aligned}
$$

which is a sampled LTI representation of the system (1).

2) Prediction Error Model: Similarly to the LTI case, the one-step-ahead prediction error can be expressed and defined as [10]:

$$
\varepsilon_{\rho}\left(t_{k}\right)=y\left(t_{k}\right)-\hat{y}_{\rho}\left(t_{k}\right),
$$

where $\hat{y}_{\rho}\left(t_{k}\right)$ is the one step ahead predictor based on the model (6). Under the commonly used assumption that noisefree observation of the sequence $\left\{p_{k}, p_{k-1}, \ldots\right\}$ is available and based on the MISO-LTI form (10), $\hat{y}_{\rho}\left(t_{k}\right)$ is defined as:

$$
\begin{aligned}
\hat{y}_{\rho}\left(t_{k}\right)=-\left(\sum_{i=1}^{n_{\mathrm{a}}} \sum_{l=1}^{n_{\alpha}} \frac{a_{i, l}}{F(d, \rho)} \chi_{i, l}\right)\left(t_{k}\right) \\
+\left(\sum_{j=0}^{n_{\mathrm{b}}} \sum_{l=0}^{n_{\beta}} \frac{b_{j, l}}{F(d, \rho)} u_{k, j}\right)\left(t_{k}\right) .
\end{aligned}
$$

3) Prediction error minimization: Denote $\mathcal{D}_{N}=$ $\left\{y\left(t_{k}\right), u\left(t_{k}\right), p\left(t_{k}\right)\right\}_{k=1}^{N}$ a data sequence of $\mathcal{S}_{\mathrm{o}}$. Then to provide an estimate of $\rho$ based on the minimization of $\varepsilon_{\rho}$, an identification criterion $W\left(\mathcal{D}_{N}, \rho\right)$ can be introduced, like the least square criterion

$$
W\left(\mathcal{D}_{N}, \rho\right)=\frac{1}{N} \sum_{k=1}^{N} \varepsilon_{\rho}^{2}\left(t_{k}\right),
$$

such that the parameter estimate is

$$
\hat{\rho}_{N}=\arg \min _{\rho \in \mathbb{R}^{n} \rho} W\left(\mathcal{D}_{N}, \rho\right) .
$$

4) CT filtering and sampled data: The output predictor (12) of the model structure consists of a "sampled" CT filtering operation, so it becomes a question how to evaluate (12) in practice when only sampled measurements of the CT signals $(y, p, u)$ are available. In order to apply a CT filter on sampled data one can either interpolate the samples to obtain a continuous-time signal and apply the CT filter on it or use a numerical approximation, i.e. DT approximation of the considered system. This is a common problem in simulation of continuous-time systems. For simulation purposes, DT approximation of the system can efficiently be dealt with by using powerful numerical algorithms available [2].

Note that to derive an accurate DT approximation of the system itself, it is often sufficient in terms of the classical discretization theory to assume that the sampled free CT signals of the system are restricted to be constant in the sampling period [6], which has also been shown in case of LPV systems with static dependence [20]. This provides the hypothesis, also used in [15], [7], that if CT $(p, u)$ are piecewise constant between two samples, then the trajectory of $y$ is completely determined by its observations at the sample period $\mathrm{T}_{\mathrm{s}} k$. Therefore, under these inter-sampling conditions, the following operation is well-defined [5]:

$$
(F(d) y)\left(t_{k}\right)=F(d) y\left(t_{k}\right),
$$

Under this assumption, and considering that a CT filter can only be applied to sampled data via numerical approximation, the usual filter properties such as commutativity holds between a DT filter and the numerical approximation of a CT filter. Nevertheless, it is important to notice that the numerical approximation method used to approximate the CT filter does not introduce any transformation of the coefficients to be estimated which remain, in terms of (12), the coefficients of the parsimonious CT model. 


\section{Identification problem statement}

Based on the previous considerations, the identification problem addressed in the sequel can now be defined.

Problem 1: Given a CT-LPV data generating system $\mathcal{S}_{\mathrm{o}}$ defined as in (1) and a data set $\mathcal{D}_{N}$ collected from $\mathcal{S}_{0}$. Based on the CT-LPV model structure $\mathcal{M}_{\rho}$ defined by (6), estimate the parameter vector $\rho$ using $\mathcal{D}_{N}$ under the following assumptions:

\section{A1 $\mathcal{S}_{\mathrm{o}} \in \mathcal{M}$.}

A2 In the parametrization (4a-b), $\left\{f_{l}\right\}_{l=1}^{n_{\alpha}}$ and $\left\{g_{l}\right\}_{l=1}^{n_{\beta}}$ are chosen such that $\left(\mathcal{G}_{0}\right)$ is identifiable for any trajectory of $p$.

A3 $u\left(t_{k}\right)$ is not correlated to $e_{\mathrm{o}}\left(t_{k}\right)$.

A4 $\mathcal{D}_{N}$ is informative with respect to $\mathcal{M}$.

A5 $\mathcal{S}_{\mathrm{o}}$ is globally BIBO stable, i.e. for any trajectory of $p: \mathbb{R} \rightarrow \mathbb{P}$ and any bounded input signal $u$, the output of $\mathcal{S}_{\mathrm{O}}$ is bounded [20].

\section{REFINED INSTRUMENTAL VARIABLE FOR LPV SYSTEMS}

Based on the MISO-LTI formulation (12), it becomes possible theoretically to achieve optimal PEM using linear regression [9]. This allows to extend the Refined Instrumental Variable (RIV) approach of the LTI identification framework to provide an efficient way of identifying CT-LPV models.

\section{A. Linear Regression for CT-LPV-BJ models}

Using the LTI model (6), reformulated as (12), $y\left(t_{k}\right)$ can be written in the regression form:

$$
y^{\left(n_{\mathrm{a}}\right)}\left(t_{k}\right)=\varphi^{\top}\left(t_{k}\right) \rho+\tilde{v}\left(t_{k}\right)
$$

where,

$$
\begin{aligned}
& \varphi\left(t_{k}\right)=\left[-y^{\left(n_{\mathrm{a}}-1\right)}\left(t_{k}\right) \ldots-y\left(t_{k}\right)-\chi_{1,1}\left(t_{k}\right) \ldots\right. \\
& \left.\ldots-\chi_{n_{\mathrm{a}}, n_{\alpha}}\left(t_{k}\right) u_{0,0}\left(t_{k}\right) \ldots u_{n_{\mathrm{b}}, n_{\beta}}\left(t_{k}\right)\right]^{\top} \\
& \rho=\left[\begin{array}{lllllllll}
a_{1,0} & \ldots & a_{n_{\mathrm{a}}, 0} & a_{1,1} & \ldots & a_{n_{\mathrm{a}}, n_{\alpha}} & b_{0,0} & \ldots & b_{n_{\mathrm{b}}, n_{\beta}}
\end{array}\right]^{\top} \\
& \tilde{v}\left(t_{k}\right)=F(d, \rho) e\left(t_{k}\right) \text {. }
\end{aligned}
$$

The extended regressor in (16) contains the noise-free output terms $\left\{\chi_{i, k}\right\}$. Therefore, by momentary assuming that $\left\{\chi_{i, l}\left(t_{k}\right)\right\}_{i=1, l=0}^{n_{\mathrm{a}}, n_{\alpha}}$ are known a priori, the prediction error $\varepsilon_{\rho}\left(t_{k}\right)$ for (16) is given in terms of (11) as:

$$
\begin{gathered}
\varepsilon_{\rho}\left(t_{k}\right)=\left(F(d, \rho) y_{\mathrm{f}}\right)\left(t_{k}\right)-\sum_{i=1}^{n_{\mathrm{a}}} \sum_{l=1}^{n_{\alpha}} a_{i, l} \chi_{i, l}^{\mathrm{f}}\left(t_{k}\right) \\
+\sum_{j=0}^{n_{\mathrm{b}}} \sum_{l=0}^{n_{\beta}} b_{j, l} u_{k, j}^{\mathrm{f}}\left(t_{k}\right),
\end{gathered}
$$

where $y_{\mathrm{f}}\left(t_{k}\right), u_{j, l}^{\mathrm{f}}\left(t_{k}\right)$ and $\chi_{i, l}^{\mathrm{f}}\left(t_{k}\right)$ represent the outputs of an hybrid prefiltering operation, involving the continuous-time filter (see [27]):

$$
Q_{\mathrm{c}}(d, \rho)=\frac{1}{F(d, \rho)}
$$

Based on (17), the associated linear-in-the-parameters model takes the form [27]:

$$
y_{\mathrm{f}}^{\left(n_{\mathrm{a}}\right)}\left(t_{k}\right)=\varphi_{\mathrm{f}}^{\top}\left(t_{k}\right) \rho+\tilde{v}_{\mathrm{f}}\left(t_{k}\right)
$$

where

$$
\begin{aligned}
& \varphi_{\mathrm{f}}\left(t_{k}\right)=\left[-y_{\mathrm{f}}^{\left(n_{\mathrm{a}}-1\right)}\left(t_{k}\right) \ldots-y_{\mathrm{f}}\left(t_{k}\right)-\chi_{1,1}^{\mathrm{f}}\left(t_{k}\right) \ldots\right. \\
& \left.\ldots-\chi_{n_{\mathrm{a}}, n_{\alpha}}^{\mathrm{f}}\left(t_{k}\right) u_{0,0}^{\mathrm{f}}\left(t_{k}\right) \ldots u_{n_{\mathrm{b}}, n_{\beta}}^{\mathrm{f}}\left(t_{k}\right)\right]^{\top} \\
& \tilde{v}_{\mathrm{f}}\left(t_{k}\right)=Q_{\mathrm{c}}(d, \rho) \tilde{v}\left(t_{k}\right)=e\left(t_{k}\right) .
\end{aligned}
$$

\section{$B$. The refined instrumental variable approach}

Under the assumption that the CT filter $Q_{\mathrm{c}}(d, \rho)$ and $\left\{\chi_{i, l}\left(t_{k}\right)\right\}_{i=1, l=0}^{n_{\mathrm{a}}, n_{\alpha}}$ are known a priori, traditional parametric estimation methods from the LTI framework could provide efficient estimates of $\rho$. However, in practice, $Q_{\mathrm{c}}(d, \rho)$ is usually unknown and can only be estimated. Furthermore, it is important to note that the regressors (19) and (16) contain time-derivatives of $y$ and $u$ which, in the assumed framework considering sampled data, can only be approximated. It is well-known that the approximation of derivatives requires low-pass filtering of the corresponding signals. The most commonly used filters for this purpose are Poisson's filters, or state-variable filters [4]. However, in the proposed approach $F(d, \rho)$ achieves this stable low-pass filtering directly. Therefore, it is a particular strength of the presented reformulation (19) that the estimated filter $F(d, \rho)$ is not only used for the minimization of the prediction error but it also provides the filtering for the approximation of the time derivatives. In order to estimate the parameter vector in (19) without the prior knowledge of $Q_{\mathrm{c}}(d, \rho)$, the RIV method is proposed due to the following reasons:

- RIV methods lead to optimal estimates in the LTI case if $\mathcal{S}_{\mathrm{o}} \in \mathcal{M}$ (see [19], [26], [27]).

- In a practical situation of identification, $\mathcal{G}_{\mathrm{o}} \in \mathcal{G}$ might be fulfilled due to first principle or expert's knowledge. However, it is commonly fair to assume that the model is not OE. In such case, RIV methods have the advantage of providing consistent estimates whereas methods such as extended least squares (LS) are biased and more advanced PEM methods need robust initialization [12].

- The RIV algorithm has been successfully used for models with similar CT structure, like in the case of linear models [16], [26] and nonlinear ones [8].

Aiming at the extension of the RIV approach for the estimation of CT LPV models, consider the relationship between the process input and output signals as in (16). Based on this form, the extended-IV estimate is given as [26]:

$$
\begin{aligned}
\hat{\rho}_{\mathrm{XIV}}(N)=\arg \min _{\rho \in \mathbb{R}^{\mathbb{N}_{\rho}}} & \|\left[\frac{1}{N} \sum_{k=1}^{N} \zeta_{\mathrm{f}}\left(t_{k}\right) \varphi_{\mathrm{f}}^{\top}\left(t_{k}\right)\right] \rho \\
- & {\left[\frac{1}{N} \sum_{t=1}^{N} \zeta_{\mathrm{f}}\left(t_{k}\right) y_{\mathrm{f}}^{\left(n_{\mathrm{a}}\right)}\left(t_{k}\right)\right] \|_{W}^{2}, }
\end{aligned}
$$

where $\zeta\left(t_{k}\right)$ is the instrument, $\|x\|_{W}^{2}=x^{T} W x$, with $W$ a positive definite weighting matrix and the filtered variables $\zeta_{\mathrm{f}}, \varphi_{\mathrm{f}}$ and $y_{\mathrm{f}}$ are constructed using a stable prefilter. If $G_{\mathrm{o}} \in$ $\mathcal{G}$, the extended-IV estimate is consistent under the following two conditions ${ }^{2}$ :

\footnotetext{
${ }^{2}$ The notation $\overline{\mathbb{E}}\{\cdot\}=\lim _{N \rightarrow \infty} \frac{1}{N} \sum_{t=1}^{N} \mathbb{E}\{$.$\} is adopted from the$ prediction error framework of [10].
} 
C1 $\overline{\mathbb{E}}\left\{\zeta_{\mathrm{f}}\left(t_{k}\right) \varphi_{\mathrm{f}}^{\top}\left(t_{k}\right)\right\}$ is full column rank.

C2 $\quad \overline{\mathbb{E}}\left\{\zeta_{\mathrm{f}}\left(t_{k}\right) \tilde{v}_{\mathrm{f}}\left(t_{k}\right)\right\}=0$.

Moreover it has been shown in [19], [25] and [26] that the minimum variance estimator can be achieved if:

C3 $W=I$.

C4 $\zeta$ is chosen as the noise-free version of the extended regressor in (16) and is therefore defined in the present LPV case as:

$$
\begin{gathered}
\zeta\left(t_{k}\right)=\left[-\chi^{\left(n_{\mathrm{a}}-1\right)}\left(t_{k}\right) \ldots-\chi\left(t_{k}\right)-\chi_{1,1}\left(t_{k}\right) \ldots\right. \\
\left.\ldots \chi_{n_{\mathrm{a}}, n_{\alpha}}\left(t_{k}\right) u_{0,0}\left(t_{k}\right) \ldots u_{n_{\mathrm{b}}, n_{\beta}}\left(t_{k}\right)\right]^{\top} .
\end{gathered}
$$

C5 $\mathcal{G}_{0} \in \mathcal{G}$ and $n_{\rho}$ is equal to the minimal number of parameters required to represent $\mathcal{G}_{\text {o }}$ with the considered model structure.

C6 The CT filter used is chosen as the filter (18).

While conditions $\mathrm{C} 1-3$ and $\mathrm{C} 5$ are quite straight-forward to fulfill (see [19], [25]), the construction of a suitable instrument and filter fulfilling $\mathrm{C} 4$ and $\mathrm{C} 6$ are not trivial in practice. The RIV algorithm involves an iterative (or relaxation) algorithm in which, at each iteration, an 'auxiliary model' is used to generate the instrumental variables (which guarantees C2), as well as the associated prefilters. This auxiliary model is based on the parameter estimates obtained at the previous iteration. Consequently, if convergence occurs, C4 and C6 are fulfilled. Thus, the RIV is a suitable method to i) efficiently estimate the parameter vector $\rho$ in (19) when $S_{\text {o }} \in \mathcal{M}$ and ii) consistently estimate $\rho$ in the practical situation when the noise model structure is inaccurate.

\section{The LPV-RIVC Algorithm}

Step 1 The usual initialization for the CT-RIV algorithm is a DT model estimate obtained by an LS method or a DT-RIV algorithm. In the LPV case however, the transformation of a DT model into a CT model is not trivial. Consequently, the initial estimate proposed for the LPV-RIVC algorithm is an LTI-RIVC estimate of $\mathcal{M}_{\rho}$, i.e. $\hat{\rho}^{(0)}$ is given. Set $\tau=0$.

Step 2 Compute an estimate of $\chi\left(t_{k}\right)$ via numerical approximation of

$$
A\left(p_{t}, d, \hat{\rho}^{(\tau)}\right) \hat{\chi}(t)=B\left(p_{t}, d, \hat{\rho}^{(\tau)}\right) u(t),
$$

where $\hat{\rho}^{(\tau)}$ is estimated in the previous iteration. Based on $\mathcal{M}_{\hat{\rho}(\tau)}$, deduce $\hat{\chi}\left(t_{k}\right)$ which is bounded according to Assumption A5.

Step 3 Compute the estimated continuous-time filter $\hat{Q}_{\mathrm{c}}\left(d, \hat{\rho}^{(\tau)}\right)=\frac{1}{F\left(d, \hat{\rho}^{(\tau)}\right)}$, where $F\left(d, \hat{\rho}^{(\tau)}\right)$ is as given in (8).

Step 4 Use the CT filter $\hat{Q}_{\mathrm{c}}\left(d, \hat{\rho}^{(\tau)}\right)$ as well as $\hat{\chi}\left(t_{k}\right)$ in order to generate the estimates of the derivatives which are needed:

Step 5 Build the filtered estimated regressor $\hat{\varphi}_{\mathrm{f}}\left(t_{k}\right)$ and, in terms of $\mathrm{C} 4$, the filtered instrument $\hat{\zeta}_{\mathrm{f}}\left(t_{k}\right)$.
Step 6 The solution of the IV optimization problem is then

$$
\begin{aligned}
& \hat{\rho}^{(\tau+1)}(N)= \\
& {\left[\sum_{k=1}^{N} \hat{\zeta}_{\mathrm{f}}\left(t_{k}\right) \hat{\varphi}_{\mathrm{f}}^{\top}\left(t_{k}\right)\right]^{-1} \sum_{k=1}^{N} \hat{\zeta}_{\mathrm{f}}\left(t_{k}\right) y_{\mathrm{f}}^{\left(n_{\mathrm{a}}\right)}\left(t_{k}\right)}
\end{aligned}
$$

Step 7 If $\rho^{(\tau+1)}$ has converged or the maximum number of iterations is reached, then stop, else increase $\tau$ by 1 and go to Step 2 .

\section{Simulation Example}

In order to demonstrate the performance of the presented algorithm, the following data-generating system is considered:

$$
\mathcal{S}_{\mathrm{o}}\left\{\begin{array}{l}
A_{\mathrm{o}}(d, p)=d^{2}+a_{1}^{\mathrm{o}}(p) d+a_{2}^{\mathrm{o}}(p) \\
B_{\mathrm{o}}(d, p)=b_{0}^{\mathrm{o}}(p) d+b_{1}^{\mathrm{o}}(p)
\end{array}\right.
$$

where

$$
\begin{array}{ll}
a_{1}^{\mathrm{o}}(p)=1-0.5 p, & a_{2}^{\mathrm{o}}(p)=5+3 p, \\
b_{0}^{\mathrm{o}}(p)=2+p, & b_{1}^{\mathrm{o}}(p)=5-p .
\end{array}
$$

By simulation of $\mathcal{S}_{\text {o }}$ with zero initial conditions, 3000 samples of data are collected in a $15 \mathrm{~s}$ simulation interval $\left(\mathrm{T}_{\mathrm{s}}=0,005 \mathrm{~s}\right)$. In the simulation, $u$ is chosen as a white noise with a uniform distribution $\mathcal{U}(-1,1)$ while the scheduling variable is taken as $p(t)=\sin \left(\frac{2}{3} \pi t\right)$.

The following model structure in terms of (3) is considered to capture the dynamics of $\mathcal{S}_{\mathrm{o}}$ :

$$
\mathcal{M}\left\{\begin{array}{l}
A(d, p)=d^{2}+a_{1}(p) d+a_{2}(p), \\
B(d, p)=b_{0}(p) d+b_{1}(p),
\end{array}\right.
$$

where

$$
\begin{array}{ll}
a_{1}(p)=a_{1,0}+a_{1,1} p, & a_{2}(p)=a_{2,0}+a_{2,1} p, \\
b_{0}(p)=b_{0,0}+b_{0,1} p, & b_{1}(p)=b_{1,0}+b_{1,1} p .
\end{array}
$$

To show the statistical performance of the proposed IV approach with this example the model is estimated using both the LPV-RIVC algorithm and the MATLAB LSQNONLIN method. The LSQNONLIN method is a nonlinear statistically optimal optimization method, but being a nonlinear method it is also sensitive to initialization [11]. Therefore, in order to put this latter method at its best, it is initialized on the true parameters.

The results of Monte Carlo simulations obtained by using the above discussed methods are presented in Table I. The statistical properties of each method are evaluated using the mean and standard deviation of the estimated parameters . These results are based on $N_{\text {run }}=100$ random realizations under a Signal-to-Noise Ratio (SNR) of $10 \mathrm{~dB}$ with:

$$
\mathrm{SNR}=10 \log \frac{P_{\chi_{\mathrm{o}}}}{P_{e_{\mathrm{o}}}},
$$

where $P_{x}$ is the power of signal $x$.

Table I shows that according to the theoretical results, the estimated parameters using the LPV-RIVC algorithm are unbiased. Moreover, the LPV-RIVC method achieves 
TABLE I

Monte CARlo Simulation For SNR $=10 \mathrm{~dB}$

\begin{tabular}{|c||c||c|c||c|c||}
\hline \multicolumn{1}{|c||}{} & \multicolumn{1}{c||}{ Method } & \multicolumn{2}{c|}{ LSQNONLIN } & \multicolumn{2}{c|}{ LPV-RIVC } \\
\hline Name & True Value & mean & st. dev. & mean & st. dev. \\
\hline$a_{1,0}$ & 1 & 1.0026 & 0.0408 & 1.0040 & 0.0421 \\
\hline$a_{1,1}$ & -0.5 & -0.5054 & 0.0707 & -0.5089 & 0.0745 \\
\hline$a_{2,0}$ & 5 & 5.0017 & 0.0698 & 5.0016 & 0.0731 \\
\hline$a_{2,1}$ & 3 & 2.9996 & 0.1278 & 2.9973 & 0.1308 \\
\hline$b_{0,0}$ & 2 & 2.0004 & 0.0298 & 1.9999 & 0.0311 \\
\hline$b_{0,1}$ & 1 & 0.9988 & 0.0550 & 0.9981 & 0.0578 \\
\hline$b_{1,0}$ & 5 & 5.0008 & 0.1469 & 5.0021 & 0.1559 \\
\hline$b_{1,1}$ & -1 & -1.0274 & 0.2670 & -1.0355 & 0.2732 \\
\hline
\end{tabular}

the same performance as the optimal LSQNONLIN method, where the latter approach uses the true parameter values as initial conditions. Consequently, the presented algorithm constitutes the first direct continuous-time method which achieves efficient minimization of the prediction error in the LPV context. It appears in this example that the empirically accepted properties of the RIV based method might also apply to the LPV case even though this cannot be proven yet. Moreover, this method does not requires any filters designed by the user to approximate time-derivatives of the measured IO signals.

\section{CONCLUSION}

The proposed approach provides the very first direct global LPV identification method that is able to give consistent estimates of LPV-IO models in continuous-time and has a low computational load. The proposed algorithm has been tested on a representative numerical simulation example and it has been shown that the procedure is robust to noise and can compete with the optimal nonlinear optimization method even in the case where the latter is initialized knowing the true parameters. Furthermore, based on a previous work on CT-LTI systems operating in closed loop, this methods opens the possibility for closed-loop CT-LPV identification.

\section{REFERENCES}

[1] P. Apkarian and P. Gahinet. A convex characterization of gainscheduled $\mathcal{H}_{\infty}$ controllers. IEEE Trans. on Automatic Control, 40(5):853-864, 1995.

[2] K. E. Atkinson. An Introduction to Numerical Analysis. John Wiley and Sons, 1989.

[3] M. Dettori and C. W. Scherer. LPV design for a CD player: An experimental evaluation of performance. In Proc. of the 40th IEEE Conf. on Decision and Control, pages 4711-4716, Orlando, Florida, USA, Dec. 2001.

[4] H. Garnier, M. Mensler, and A. Richard. Continuous-time model identification from sampled data: implementation issues and performance evaluation. International Journal of Control, 76(13):1337-1357, 2003.

[5] H. Garnier and L. Wang, editors. Identification of Continuous-time Models from Sampled Data. Springer-Verlag, 2008.

[6] G. C. Goodwin, S. F. Graebe, and M. E. Salgado. Control System Design. Prentice Hall, 2000.

[7] R. Johansson. Identification of Continuous-Time models. IEEE Transactions on Signal Processing, 42(4):3887-897, 1994.

[8] V. Laurain, M. Gilson, H. Garnier, and P. C. Young. Refined instrumental variable methods for identification of Hammerstein continuoustime Box-Jenkins models. In Proc. of the 47th IEEE Conf. on Decision and Control, pages 1386-1391, Cancun, Mexico, Dec. 2008.
[9] V. Laurain, M. Gilson, R. Tóth, and H. Garnier. Refined instrumental variable methods for identification of LPV Box-Jenkins models. Automatica, 46(6):959-967, 2010.

[10] L. Ljung. System Identification, theory for the user. Prentice Hall, 1999.

[11] L. Ljung. Initialisation aspects for subspace and output-error identification methods. In European Control Conference, Cambridge, UK, Sept. 2003.

[12] L. Ljung. Experiments with identification of continuous time models. In Proceedings of the 15th IFAC Symposium on System Identification, Saint-Malo, France, July 2009.

[13] A. Marcos and G. J. Balas. Development of linear-parameter-varying models for aircraft. Journal of Guidance, Control and Dynamics, 27(2):218-228, 2004

[14] K. Z. Østergaard, J. Stousturp, and P. Barth. Rate bounded linear parameter varying control of a wind turbine in full load operation. In Proc. of the 17th IFAC World Congress, Seoul, Korea, July 2008.

[15] R. Pintelon, J. Schoukens, and Y. Rolain. Box-Jenkins continuous-time modeling. Automatica, 36(7):983-991, 2000.

[16] G. P. Rao and H. Garnier. Numerical illustrations of the relevance of direct continuous-time model identification. In Proceedings of the 15th IFAC World Congress on Automatic Control, Barcelona, Spain, 2002.

[17] G. P. Rao and H. Unbehauen. Identification of continuous-time systems: direct or indirect ? Systems Science, 30(3):25-50, 2004

[18] C. W. Scherer. Mixed $\mathcal{H}_{2} / \mathcal{H}_{\infty}$ control for time-varying and linear parametrically-varying systems. Int. Journal of Robust and Nonlinear Control, 6(9-10):929-952, 1996.

[19] T. Söderström and P. Stoica. Instrumental Variable Methods for System Identification. Springer-Verlag, New York, 1983.

[20] R. Tóth. Modeling and Identification of Linear Parameter-Varying Systems. Lecture Notes in Control and Information Sciences, Vol. 403. Springer-Germany, 2010.

[21] R. Tóth, P. S. C. Heuberger, and P. M. J. Van den Hof. On the discretization of LPV state-space representations. IET Control Theory \& Applications, 4:2082-2096, 2010.

[22] R. Tóth, M. Lovera, P. S. C. Heuberger, and P. M. J. Van den Hof. Discretization of linear fractional representations of LPV systems. In Proc. of the 48th IEEE Conf. on Decision and Control, pages 7424 7429, Shanghai, China, Dec. 2009

[23] F. Wijnheijmer, G. Naus, W. Post, M. Steinbuch, and P. Teerhuis Modeling and LPV control of an electro-hydraulic servo system. In Proc. of the IEEE International Conf. on Control Applications, pages 3116-3120, Munich, Germany, Oct. 2006.

[24] F. Wu and K. Dong. Gain-scheduling control of LFT systems using parameter-dependent Lyapunov functions. Automatica, 42(1):39-50, 2006

[25] P. C. Young. Recursive Estimation and Time-Series Analysis. SpringerVerlag, Berlin, 1984.

[26] P. C. Young. The refined instrumental variable method: Unified estimation of discrete and continuous-time transfer function models. Journal Européen des Systèmes Automatisés, 42:149-179, 2008.

[27] P. C. Young, H. Garnier, and M. Gilson. Identification of continuoustime models from sampled data. In H. Garnier and L. Wang, editors, Refined instrumental variable identification of continous-time hybrid Box-Jenkins models., pages 91-131. Springer-Verlag, London, 2008.

[28] K. Zhou and J. C. Doyle. Essentials of Robust Control. Prentice-Hall, 1998. 